Supporting Information

\title{
On the Maximal Output Energy Density of Nanogenerators
}

Jingjing Fu ${ }^{a, b}$, Xin Xia ${ }^{a}$, Guoqiang Xu ${ }^{a}$, Xiaoyi Li ${ }^{a}$, Yunlong Zi ${ }^{a, b,{ }^{*}}$

${ }^{a}$ Department of Mechanical and Automation Engineering, The Chinese University of Hong Kong, Shatin, N.T., Hong Kong SAR, China

${ }^{b}$ Shun Hing Institute of Advanced Engineering, The Chinese University of Hong Kong, Shatin, N.T., Hong Kong SAR, China

*Corresponding author: ylzi@,cuhk.edu.hk

KEYWORDS: maximal output energy density; triboelectric nanogenerator; breakdown effect; piezoelectric nanogenerator; electromagnetic generator. 

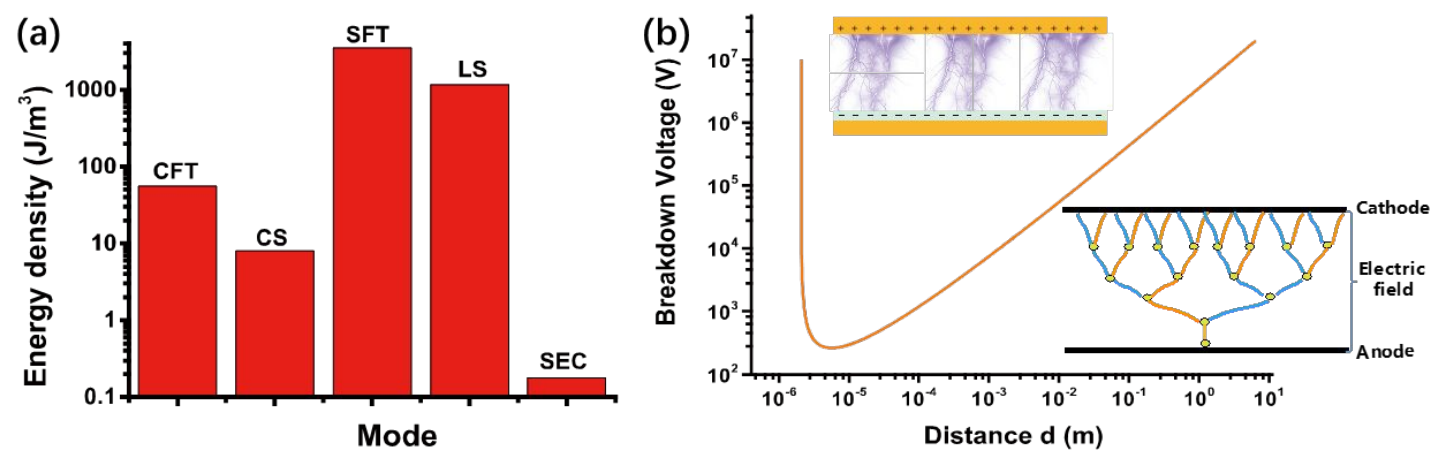

Figure S1. The energy density and breakdown phenomenon in TENG. (a) The energy density of different modes of TENG. (b)The breakdown model of contactseparation-trigged TENG, in which the insert is the Townsend discharging model for explaining the breakdown phenomena for this mode, the curve is the breakdown voltage calculated by Paschen's law, at 1 atm. 

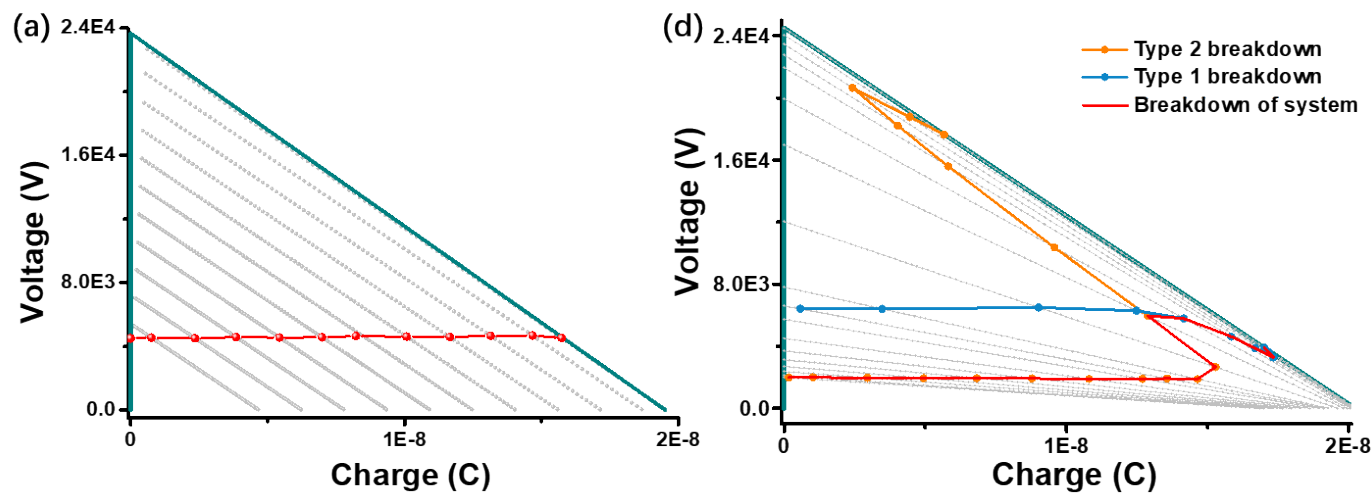

Figure S2. The method to mark the critical breakdown voltage and transferred charge in $Q-V$ curve for CMEO cycle. (a) The $Q-V$ curve of SFT with a dielectric moving part, in which the green wireframe is the max energy per cycle, the gray points represent the voltage and transferred charge, from short-circuit status to open-circuit status, and the red point-to-point line is the critical breakdown voltage and transferred charge. (b) The $Q-V$ curve of SFT with a metal moving part, in which the blue and orange point-to-point line are the critical breakdown voltage and transferred charge of the type 1 and type 2 breakdown, respectively. As for the meaning of the green wireframe, the gray points and the red point-to-point line, they are just same with that in Figure S1 a. 

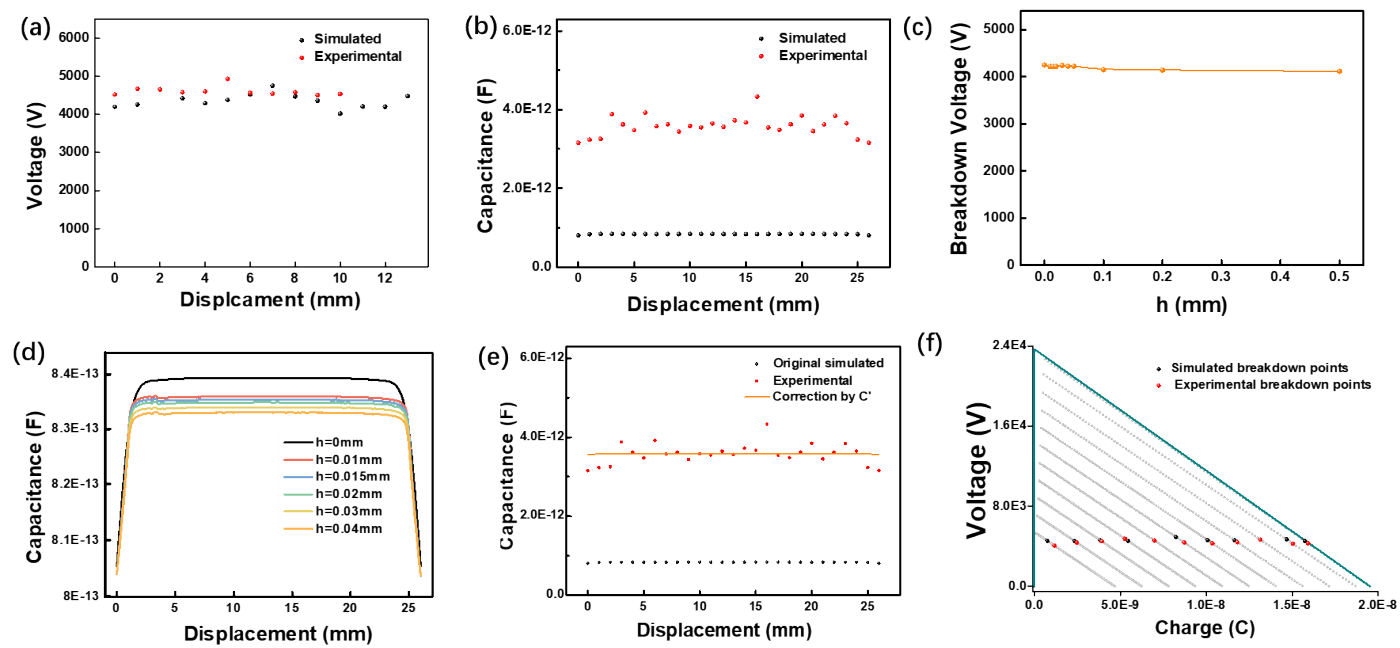

Figure S3. The correction of simulation for SFT TENG with a dielectric moving

part. (a) and (b) The comparison of the breakdown voltage and capacitance of experimental result red with the simulated black. (c) and (d) The breakdown voltage and capacitance of TENG at a different air gap, $h$, between the top moving part and the bottom static part. (e) The capacitance result corrected by parasitic capacitor. (f) The method to point the experimental data in simulated $Q-V$ curve for CMEO. 
(a)

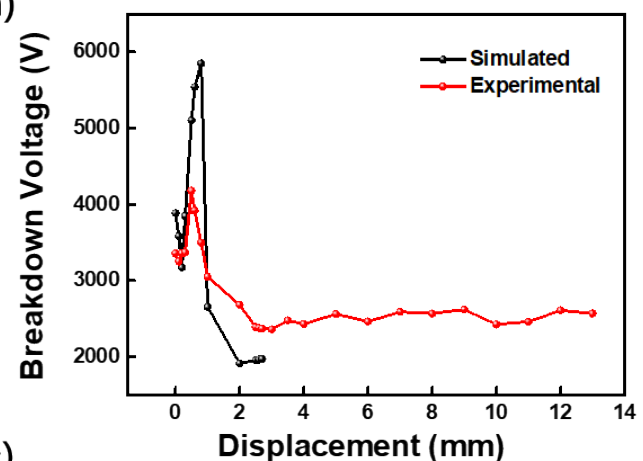

(c)

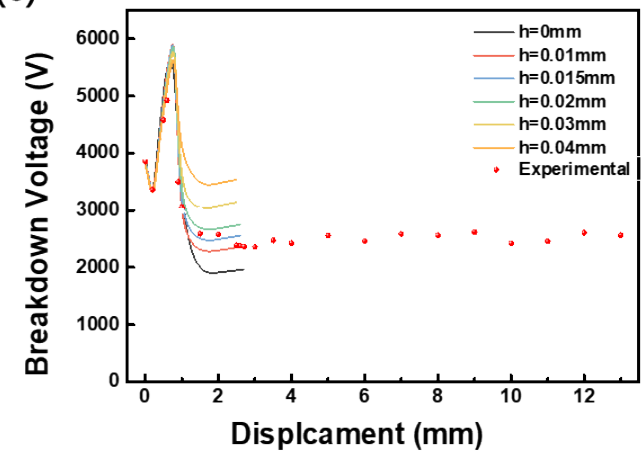

(b)

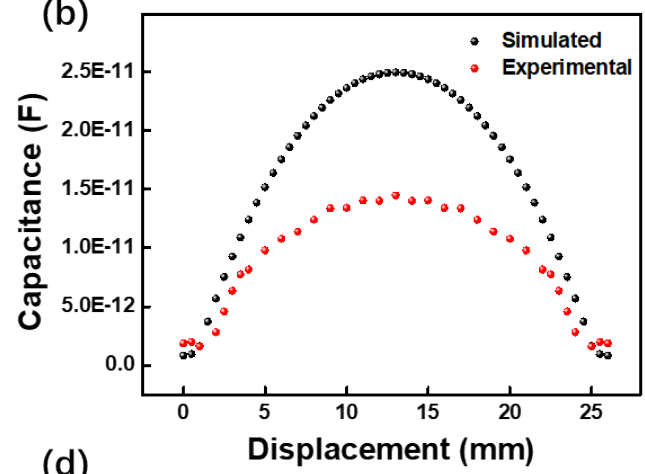

(d)

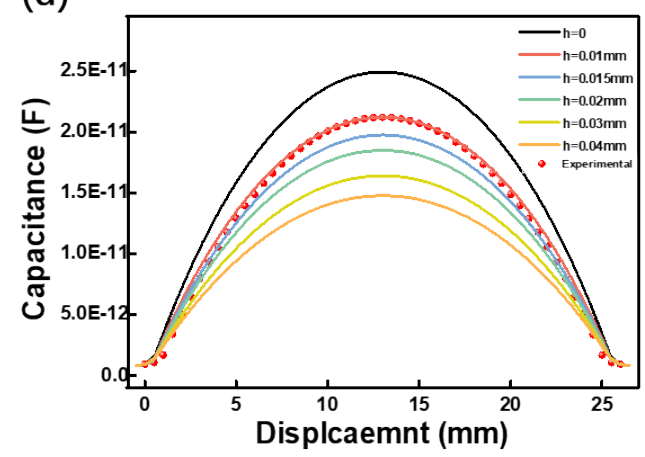

Figure S4. The correction of simulation for SFT TENG with a metal moving part.

(a) and (b) The comparison of the breakdown voltage and capacitance of experimental result red with the simulated black. (c) The breakdown voltage of SFT with different air gap, $h$, compared with the experimental result. (d) The capacitance of SFT with different air gap, $h$, compared with the experimental result. 
(a)
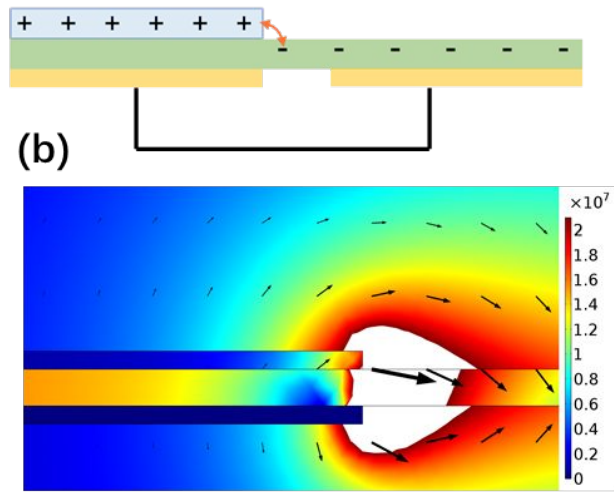

(c)

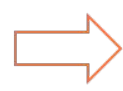

(d)
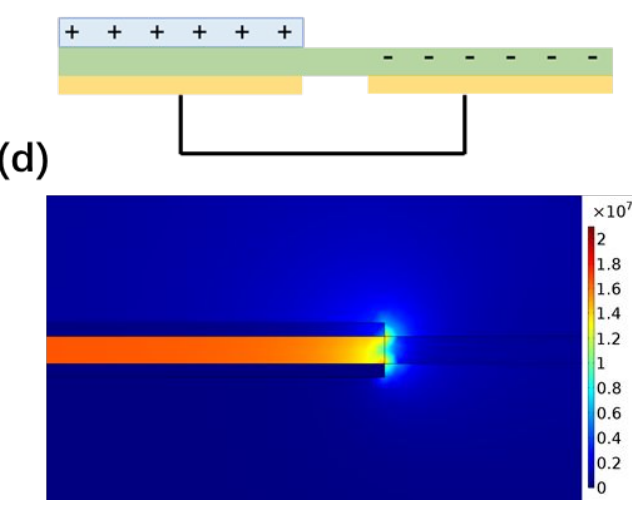

Figure S5. The correction of the module under the high charge density. (a) and (b)

show the initial module for SFT with a dielectric moving part. (c) and (d) are corrected module. All modules are at short circuit state, $\sigma=300 \mu \mathrm{C} . \mathrm{m}^{-2}$. 

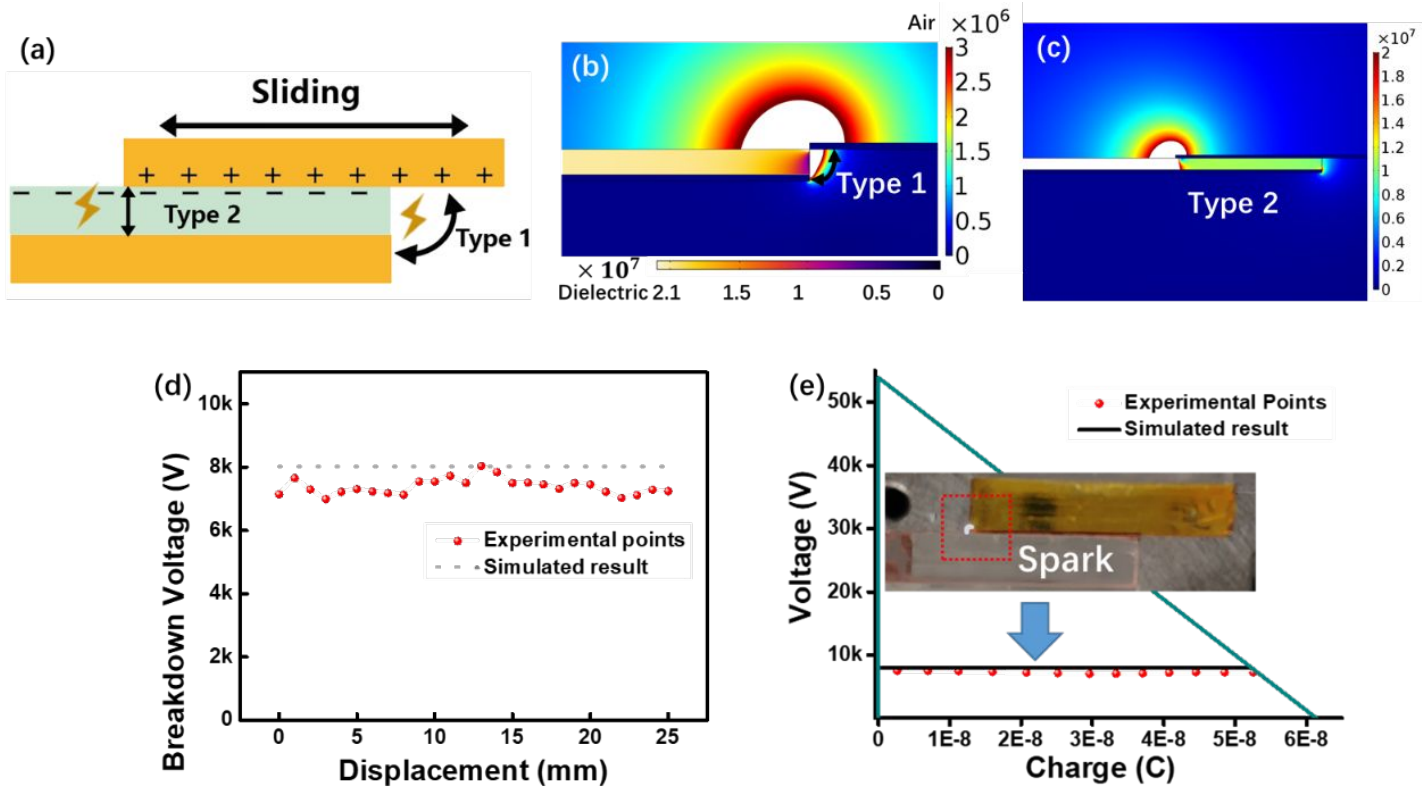

Figure S6. The simulation of the breakdown of LS mode TENG. (a) The model diagram of LS mode TENG. (b) The electric field distribution showing type 1 breakdown (in air). (c) The electric field distribution showing type 2 breakdown (in the dielectric layer). (d) and (e) compare the experimental and simulated results of the breakdown points in LS-TENG with charge density $\sigma=100 \mu \mathrm{C} / \mathrm{m}^{2}$, respectively, while only type 1 breakdown exists. The insert is a picture showing a spark. 
Supporting Table 1. The size parameters

\begin{tabular}{|c|c|c|c|}
\hline $\begin{array}{l}\text { Mode } \\
\text { Parts }\end{array}$ & SFT-dielectric & SFT-metal & $\mathbf{L S}$ \\
\hline $\begin{array}{l}\text { Electrodes } \\
(\mathrm{Cu})\end{array}$ & \multicolumn{3}{|c|}{$25 \mathrm{~mm} \times 25 \mathrm{~mm} \times 0.1 \mathrm{~mm}$} \\
\hline $\begin{array}{l}\text { Dielectric } \\
\text { layer } \\
(\mathrm{PDMS})\end{array}$ & \multicolumn{2}{|c|}{$54 \mathrm{~mm} \times 25 \mathrm{~mm} \times 0.4 \mathrm{~mm}$} & $25 \mathrm{~mm} \times 25 \mathrm{~mm} \times 0.4 \mathrm{~m}$ \\
\hline $\begin{array}{l}\text { Moving part } \\
\text { (Cu/Nylon) }\end{array}$ & \multicolumn{3}{|c|}{$25 \mathrm{~mm} \times 25 \mathrm{~mm} \times 0.1 \mathrm{~mm}$} \\
\hline
\end{tabular}


Note S1. The method to calculate the energy volume density of different modes of TENG.

The definition of energy volume density is always used in the energy field to describe the device's ability to store and output energy. Here we also introduce in TENG. The equation is

$$
U=\frac{E_{m}}{V_{L}}
$$

where $E_{m}$ is the maximal output energy per cycle of TENG, $V_{L}$ is the volume of the device. According to this definition, we calculated the energy density of different modes of TENG. In order to be unified and contrasted with previous standards, we used the structural figure-of-merits (FOMs) as the basic data, which has been calculated before by Zi. ${ }^{1}$ The FOMs was calculated by the equation:

$$
\mathrm{FOM}_{s}=\frac{2 \varepsilon_{0} \quad E_{m}}{\sigma^{2} A x_{\text {max }}}
$$

Here, $\varepsilon_{0}, \sigma, A$ and $x_{\max }$ all parameters given by authors. Therefore, we can calculate the maximal output energy per cycle, $E_{m}$. The structural parameters are also shown in the paper. We can get the energy density easily through this process., as shown in Figure S1a. It should be noticed that this $E_{m}$ is maximal energy per cycle without considering the effect of breakdown. 
Note S2. The method to mark the critical breakdown voltage and transferred charge in $Q-V$ curve for CMEO cycle.

In Figure $\mathrm{S} 1$, in order to plot the $Q-V$ curve of SFT TENG, firstly, we fixed the displacement of the top moving part at a certain value, then through adding the floating potential with same value but opposite polarity to two electrodes. The value of this floating potential changes from 0 to maximal short-circuit charge, representing the TENG's working state change from open circuit to short circuit. Through comparing the electric field of TENG at each state with the critical breakdown condition of materials, so that we can figure out the breakdown voltage and charge for this displacement. Using the same method, we can get the breakdown points for every displacement. Just as Figure S1, the grey points show the all voltage and charge of each displacement from open-circuit to short-circuit conditions. The red points are breakdown points for TENG. But it should be noticed that, because there is more than one type of breakdown for SFT TENG with a metal moving part, therefore, we calculated the threshold value for each breakdown mode separately, and plot them in same $Q-V$ curve, as shown in $\mathrm{S} 1 \mathrm{~b}$. The lower threshold will be the breakdown threshold of the system, and its corresponding breakdown type will be the type of the system.

Note S3. The correction of simulation for SFT TENG with a dielectric moving part.

Figure $\mathrm{S} 2 \mathrm{a}$ and $\mathrm{b}$ show the experimental result comparing with the simulated result. We can notice that, for the breakdown voltage, there is no obvious difference between the 
experimental result and simulated one, as shown in S2a. But when focusing on the capacitance, there still exists an obvious difference. For fitting with experimental better, we corrected the simulation model from two aspects. First is the air gap, $h$, between the top moving part and bottom static part. Second is the parasitic capacitor from the surrounding and experimental equipment. In practical application, even if pushing the mass on the top of TENG, the air gap between the top and bottom two parts can hardly be ignored, due to the imperfect surface of the material. But according to the simulation result with correction of the air gap, $h$, we found that the breakdown voltage, S2c, and capacitance, S2c, of SFT with a dielectric top moving part don't change too much with the air gap, $h$. The reason for this phenomenon is that the polarization of the dielectric layer is weak, and the change in position changes the distribution of the electric field. The parasitic capacitor is also an important factor cannot be ignored and avoided. S2b show different between experimental result and simulated one. Because cannot directly calculated the parasitic from existing theory, we calculate the difference between experimental and theoretical results at various multi-displacement many times, and finally figure out the value of the parasitic capacitance in this experiment is about about $2.8 \times 10^{-12} \mathrm{~F}$. And correcting the simulation result with this value, as shown in Figure S2e. Finnaly according the corrected result, plot the experimental result and simulated result in same $Q-V$ curve.

\section{Note S4. The correction of simulation for SFT TENG with a metal moving part.}


$\mathrm{S} 3 \mathrm{a}$ and $\mathrm{b}$ show the experimental result with simulated one. There existing an obvious difference, especially the medium part. We can notice that the capacitance is metalTENG at $10^{-11} \mathrm{~F}$ level, so the effect of parasitic capacitor can be ignored. The air gap between top and bottom part is main reason for difference of the breakdown voltage and capacitance. So, we calculated the result with different $h$ and plot them with the experimental data in same figure S3c and S3d. Through compared with each other, we find that the simulation result with $h=0.01 \mathrm{~mm}$ fits with the experimental date very well. So, we could use this $\mathrm{h}$ as the correction factor for our simulation.

\section{Note S5. The simulation of SFT-dielectric, SFT-metal and LS TENG under different charge density}

The simulation was carried out by 2 -d dimensional stationary electrostatic package. The size parameters used for these three different modes were mainly keeping same, as shown in Supporting Table 1. Instead of the first cycle, the stable motion state is more meaningful for our research. Instead of the first moving cycle, the output performance of the stable motion states is more meaningful for our research. The electrostatic assignment of mode is totally same with before, just letting two electrification layers with same value but reverse polarity charge. But there are two things worth noticed: 1 . The correction for SFT TENG at a high charge density; 2. The analyses for the breakdown of LS mode.

In Figure S4, we can notice that when the charge density is high, there will exist an area 
over the threshold of the electric field. However, this kind of breakdown area isn't like the dielectric breakdown as shown in Figure 2d, in which the white area is at the overlapped part and over through the dielectric layer. The reason for this is that this type of breakdown happens between the edge of the moving part and the top surface of the middle dielectric layer. When the gap is larger and the charge density is higher, this type of breakdown is more obvious. One important thing should be noted is although this type of breakdown can hardly be avoided, it doesn't have too much influence on output performance, compared with two previously researched types of breakdown. Because this type of breakdown can only release the local charge for a short time. When the top part moves forward, the new triboelectric charge will be generated, and the released charge will be filled up again. So, in order to avoid this influence of this type of breakdown, the middle part of the sliding surface will not be added any charge density. The corrected model as shown in S4c and S4d, which can succeed to avoid the influence of this breakdown.

As shown in Figure S5, LS mode also has two types of breakdown. Type 1 is air breakdown between two electrodes, due to the edge effect. Type 2 is dielectric breakdown of middle dielectric layer. But, for the type 1 breakdown, it can be easily avoided through adding some medium or filler with high breakdown strength. So here we only consider the type 2 breakdown. And we also did the experiment to prove the simulated result as shown in Figure S5d and e. 
Note S6. The calculation of the energy density of PENG at optimal parameters and EMG at the rated rotational speed $(25 \mathrm{~Hz})$ and the low frequency $(1 \mathrm{~Hz})$.

According the newest papers, ${ }^{2,3}$ researchers have pushed the power density $\left(P_{D}\right)$ of PENG to $30 \mathrm{~mW} / \mathrm{cm}^{3}$ at $46 \mathrm{~Hz}$ and $26.4 \mathrm{~mW} / \mathrm{cm}^{3}$ at $38.89 \mathrm{~Hz}^{2}$ In order to be consistent with the energy density of TENG, we only consider the energy density within one cycle. The formula is as follows:

$$
U=\frac{P_{D}}{f}
$$

We get that $U_{(46 \mathrm{~Hz})}=652 \mathrm{~J} / \mathrm{m}^{3}$ and $U_{(38.89 \mathrm{~Hz})}=691.695 \mathrm{~J} / \mathrm{m}^{3}$, therefore we used the data of $U_{(38.89 H z)}{ }^{2}$ in this paper, although the $P_{D}(46 \mathrm{~Hz})>P_{D}(38.89 \mathrm{~Hz})$.

EMG talked in this paper is a commercial electromagnetic generator (INFINITY GM 10) made by INFINITY SAV https://infinitysav.com/magneticgenerator/. The effective power output $(P)$ is $10 \mathrm{~kW}$ when motor rotation $(\omega)$ is at $1500 \mathrm{rpm}$. The contractual parameter of this EMG is $750 \times 715 \times 528 \mathrm{~mm}$. The formula is as follows:

$$
\mathrm{U}(25 \mathrm{~Hz})=\frac{P}{f \times V}
$$

Here, $f$ is the frequency can be calculated by $\omega, f=25 \mathrm{~Hz} . V$ is the volume of this EMG. The calculated result is $U=1413.4 \mathrm{~J} / \mathrm{m}^{3}$, which means the energy density of EMG at rated frequency.

In addition to the energy density at rated frequency, we also want to know the 
performance of EMG at low frequency. Thence we further estimated the roughly energy density of EMG at $1 \mathrm{~Hz}$, according to the quadratic relationship between the power and the frequenc:

$$
\begin{gathered}
P_{o p t}^{E M G} \propto f^{2} / P_{o p t}^{E M G}=k f^{2} \\
P_{O P T}^{E M G}(1 H z)=\frac{P_{O P T}^{E M G}(25 H z)}{f(25 H z)^{2}} \times f(1 H z)^{2}
\end{gathered}
$$

Put this value, $P_{O P T}^{E M G}(1 \mathrm{~Hz})=3.2 \mathrm{~W}$, into the formula for calculating energy density, and we can get the energy density:

$$
U(1 H z)=\frac{P_{O P T}^{E M G}(1 H z)}{f(1 H z) \times V}
$$

After calculating, we can notice that the energy density at $1 \mathrm{~Hz}$ is equal to $0.4521 \mathrm{~J} / \mathrm{m}^{3}$, which is much smaller than that at $25 \mathrm{~Hz}$ and that of TENG.

REFERENCE (Only for Supporting Information)

1. Zi, Y.; Niu, S.; Wang, J.; Wen, Z.; Tang, W.; Wang, Z. L., Standards and Figureof-Merits for Quantifying the Performance of Triboelectric Nanogenerators. Nat. Commun. 2015, 6, 8376.

2. Dong, X.; Yi, Z.; Kong, L.; Tian, Y.; Liu, J.; Yang, B., Design, Fabrication, and Characterization of Bimorph Micromachined Harvester with Asymmetrical PZT Films. J. Microelectromech. Syst. 2019, 28, 700-706.

3. Hu, Y.; Yi, Z.; Dong, X.; Mou, F.; Tian, Y.; Yang, Q.; Yang, B.; Liu, J., High Power Density Energy Harvester with Non-Uniform Cantilever Structure Due to High Average Strain Distribution. Energy 2019, 169, 294-304. 